\title{
Editorial: 2D-Layered Nanomaterials: Chemical Functionalization, Advanced Characterization, and Tribological Properties
}

\author{
Andreas Rosenkranz ${ }^{1 *}$, Carsten Gachot ${ }^{2}$ and Ali Erdemir ${ }^{3}$ \\ ${ }^{1}$ Department of Chemical Engineering, Biotechnology and Materials, University of Chile, Santiago, Chile, ${ }^{2}$ Department of \\ Mechanical and Industrial Engineering, Design Engineering and Product Development, TU Wien, Vienna, Austria, ${ }^{3}$ Department of \\ Mechanical Engineering, Texas A\&M University, College Station, TX, United States
}

Keywords: 2D nanomaterials, MXenes, chemical functionalization, friction, materials characerization

Editorial on the Research Topic

2D-Layered Nanomaterials: Chemical Functionalization, Advanced Characterization, and Tribological Properties

Nowadays, a significant amount of the global energy output is consumed to overcome friction- and wear-induced problems or even damages, a situation that urgently calls for more efficient tribological solutions and strategies (Holmberg and Erdemir, 2017; Holmberg and Erdemir, 2019; Shah et al., 2020). 2D-layered nanomaterials such as graphene, graphene oxide (GO), $\mathrm{MoS}_{2}$, etc., have gained substantial attention for their use as solid lubricants and lubricant additives thus serving as promising alternatives to conventional lubrication approaches under harsh operating conditions (Berman et al., 2018; Zhang et al., 2019; Rosenkranz et al., 2020). Since 2011, the class of 2D nanomaterials has been significantly expanded by the discovery of a new class of $2 \mathrm{D}$ transition metal carbides and/or carbonitrides (Naguib et al., 2011; Naguib et al., 2014). These newly emerging nanomaterials, with $\mathrm{Ti}_{3} \mathrm{C}_{2} \mathrm{~T}_{\mathrm{x}}$ as their most prominent member, are called MXenes due to their origin in MAX-phases and their structural similarity to graphene. MXene nano-sheets have been extensively studied and also used in energy storage, catalysis, and water purification (Anasori et al., 2017; Gogotsi and Anasori, 2019; Naguib et al., 2021). During the last 2 years, MXenes have experienced increasingly more attention within the tribological community due to their remarkable solid lubrication abilities and outstanding anti-wear performance (Malaki and Varma, 2020; Marian et al., 2020; Wyatt et al., 2021).

Although they present a new paradigm as promising alternatives to conventional lubrication approaches, 2D-layered nanomaterials as solid lubricants and lubricant additives have still some shortcomings that need to be overcome to further optimize their friction and wear performance (Berman et al., 2018; Zhang et al., 2019; Rosenkranz et al., 2020). Concerning solid lubricants, the adhesion strength of the deposited nanomaterials/nano-films to the substrate is critical for the resulting tribological performance (Malaki and Varma, 2020). Regarding lubricant additives, the generally hydrophilic character of these nanomaterials lowers their dispersibility in hydrophobic oils, which goes hand in hand with stability and sedimentation problems over time. Irrespective of the nanomaterial and the application (solid lubricant or lubricant additive), the surface chemistry of these nanomaterials is considered as the key factor to solve these problems. The existing surface terminations can be used to chemically functionalize the 2D-layered nanomaterials, thus allowing for enhanced adhesion strength and improved dispersibility. Chemical modifications need to be complemented by advanced, high-resolution materials characterization (chemical and structural) 
to ensure the success of the functionalization process. Moreover, materials characterization is of utmost importance for shedding light onto the very complex nature of tribologically involved interfaces prior to and after the tribological experiments, to understand their underlying friction and wear mechanisms.

In the framework of this special issue, there are several highly innovative manuscripts ranging from new exfoliation approaches, nanoscale humidity-dependent lubrication mechanisms to the performance of hybrid nanocomposites. In this context, Zhang et al. presented a new exfoliation method for $\mathrm{MoS}_{2}$, which results in nano-flakes with bigger sizes and increased reaction yields. Claerbout et al. numerically investigated the frictional properties of multilayer $\mathrm{MoS}_{2}$ during sliding in the presence of water by nonequilibrium molecular dynamics simulations. Zhu et al. addressed the contribution of four oxygenated acids to oxygen-containing functional groups in Hummers' method using first principle methods. Li et al. studied the friction behavior of graphene in air and nitrogen atmosphere thus considering the microstructural evolution caused by the

\section{REFERENCES}

Anasori, B., Lukatskaya, M. R., and Gogotsi, Y. (2017). 2D Metal Carbides and Nitrides (MXenes) for Energy Storage. Nat. Rev. Mater. 2 (2), 1-17. doi:10. 1038/natrevmats.2016.98

Berman, D., Erdemir, A., and Sumant, A. V. (2018). Approaches for Achieving Superlubricity in Two-Dimensional Materials. ACS nano 12 (3), 2122-2137. doi:10.1021/acsnano.7b09046

Gogotsi, Y., and Anasori, B. (2019). The Rise of MXenes. ACS nano 13 (8), 8491-8494. doi:10.1021/acsnano.9b06394

Holmberg, K., and Erdemir, A. (2017). Influence of Tribology on Global Energy Consumption, Costs and Emissions. Friction 5 (3), 263-284. doi:10.1007/ s40544-017-0183-5

Holmberg, K., and Erdemir, A. (2019). The Impact of Tribology on Energy Use and CO2 Emission Globally and in Combustion Engine and Electric Cars. Tribology Int. 135, 389-396. doi:10.1016/j.triboint.2019.03.024

Malaki, M., and Varma, R. S. (2020). Mechanotribological Aspects of MXeneReinforced Nanocomposites. Adv. Mater. 32 (38), 2003154. doi:10.1002/adma. 202003154

Marian, M., Tremmel, S., Wartzack, S., Song, G., Wang, B., Yu, J., et al. (2020). Mxene Nanosheets as an Emerging Solid Lubricant for Machine Elements towards Increased Energy Efficiency and Service Life. Appl. Surf. Sci. 523, 146503. doi:10.1016/j.apsusc.2020.146503

Naguib, M., Barsoum, M. W., and Gogotsi, Y. (2021). Ten Years of Progress in the Synthesis and Development of MXenes. Adv. Mater. 33 (39), 2103393. doi:10. 1002/adma.202103393

Naguib, M., Kurtoglu, M., Presser, V., Lu, J., Niu, J., Heon, M., et al. (2011). TwoDimensional Nanocrystals Produced by Exfoliation of Ti3AlC2. Adv. Mater. 23 (37), 4248-4253. doi:10.1002/adma.201102306

Naguib, M., Mochalin, V. N., Barsoum, M. W., and Gogotsi, Y. (2014). 25th Anniversary Article: MXenes: A New Family of Two- variation of test environments and their effects on the coefficient of coefficient. Regarding composite materials, Jakubczak et al. verified the performance of graphene-based nano-hybrids, while Chen et al. addressed the tribological performance of selfassembled multilayer films of (GO/PDDA)n.

At this point, we would like to express our sincere thanks to Frontiers Publishing Group for providing the opportunity for this special issue. We would also thank the authors of each chapter for their great contributions. Lastly, we would also thank the readers for their interest in our special issue. Undoubtedly, they will appreciate the importance of $2 \mathrm{D}$ materials in combatting friction and wear although there remain several more challenges to overcome for large-scale industrial applications.

\section{AUTHOR CONTRIBUTIONS}

All authors listed have made a substantial, direct, and intellectual contribution to the work and approved it for publication.

Dimensional Materials. Adv. Mater. 26 (7), 992-1005. doi:10.1002/ adma.201304138

Rosenkranz, A., Yangqin, L., Lin, Y., and Lei, C. (2020). 2D Nanomaterials beyond Graphene: from Synthesis to Tribological Studies. Appl. Nanoscience 2020, 1-36.

Shah, R., Woydt, M., Huq, N., and Rosenkranz, A. (2020). Tribology Meets Sustainability. Ind. Lubrication Tribology 73, 430. doi:10.1108/ILT-092020-0356

Wyatt, B. C., Rosenkranz, A., and Anasori, B. (2021). 2D MXenes: Tunable Mechanical and Tribological Properties. Adv. Mater. 33 (17), 2007973. doi:10.1002/adma.202007973

Zhang, S., Ma, T., Erdemir, A., and Li, Q. (2019). Tribology of Two-Dimensional Materials: From Mechanisms to Modulating Strategies. Mater. Today 26, 67-86. doi:10.1016/j.mattod.2018.12.002

Conflict of Interest: The authors declare that the research was conducted in the absence of any commercial or financial relationships that could be construed as a potential conflict of interest.

Publisher's Note: All claims expressed in this article are solely those of the authors and do not necessarily represent those of their affiliated organizations, or those of the publisher, the editors and the reviewers. Any product that may be evaluated in this article, or claim that may be made by its manufacturer, is not guaranteed or endorsed by the publisher.

Copyright (C) 2022 Rosenkranz, Gachot and Erdemir. This is an open-access article distributed under the terms of the Creative Commons Attribution License (CC BY). The use, distribution or reproduction in other forums is permitted, provided the original author(s) and the copyright owner(s) are credited and that the original publication in this journal is cited, in accordance with accepted academic practice. No use, distribution or reproduction is permitted which does not comply with these terms. 